\title{
A versatile click chemistry-based approach for functionalizing biomaterials of diverse nature with bioactive peptides
}

Received 00th January 20xx, Accepted 00th January 20xx

\author{
Helena Martin-Gómez ${ }^{a, b}$, Lluís Oliver-Cervellóa,b, Iván Sánchez-Campillo ${ }^{a, c}$, Vicente Marchán ${ }^{c}$ \\ Maria-Pau Ginebraa,b,d , Carlos Mas-Moruno*,a,b
}

DOI: $10.1039 / x 0 x \times 00000 x$

A novel and versatile toolkit approach for the functionalization of biomaterials of different nature is described. This methodology is based on the solid-phase conjugation of specific anchoring units onto a resin-bound azido-functionalized peptide by using click chemistry. A synergistic multifunctional peptidic scaffold with cell adhesive properties was used as a model compound to showcase the versatility of this new approach. Titanium, gold and polylactic acid surfaces were selectively biofunctionalized with this method, as validated by physicochemical surface characterization with XPS. In vitro assays using mesenchymal stem cells showed enhanced cell adhesion on the functionalized samples, proving the capacity of this strategy to efficiently bioactivate different types of biomaterials.

The optimal replacement and regeneration of nonfunctional tissues represents a recurrent challenge in our society, which is further aggravated by the increase in life expectancy and age-related diseases experienced over the last decades. To address this issue, biomaterials have emerged and evolved as powerful tools to support and promote the growth of healthy tissues. ${ }^{1,2}$ However, many synthetic materials, albeit biocompatible and mechanically adequate, lack of bioactivity and require the incorporation of biological cues to achieve the desired functions. . $^{3,4}$ To this end, a well-established strategy consist in recreating the healing microenvironment, i.e. the extracellular matrix (ECM), on the biomaterial surface by integrating naturally occurring cell adhesive molecules ${ }^{5}$ and growth factors. ${ }^{6}$ In this context, synthetic peptides are regarded as valuable molecules to functionalize the surface of materials, due to their well-defined structures, ease of synthesis, higher stability and lower risk of adverse reactions, compared to native ECM proteins. 7,8 The attachment of these molecules to the surface is commonly carried out by either simple physical adsorption or by using chemical methods like silanization. ${ }^{9-12}$

\footnotetext{
a. Department of Materials Science and Engineering, Biomaterials, Biomechanics and Tissue Engineering Group (BBT), Universitat Politècnica de Catalunya.

b. Barcelona Research Center in Multiscale Science and Engineering, Universitat Politècnica de Catalunya.

c. Departament de Química Inorgànica i Orgànica, Secció de Química Orgànica, $I B U B$, Universitat de Barcelona, Barcelona, Spain.

d. Institute for Bioengineering of Catalonia, Barcelona, Spain

tElectronic Supplementary Information (ESI) available: [details of any supplementary information available should be included here]. See DOI: $10.1039 / \mathrm{x} 0 \times x \times 0000 \mathrm{x}$
}

Alternatively, biomolecule grafting can be achieved by incorporating into the peptidic structure chemical anchors with specific affinity for a substrate. ${ }^{12-14}$ Well-known examples include the use of thiols to bind gold 15,16 or catechol groups to bind titanium and other metallic oxides. ${ }^{17,18}$ This strategy allows for a rapid, specific and efficient surface functionalization without the need of any pre-treatment of the material. However, this approach is rather restrictive since it implies that each synthesized peptide can be used only for a narrow range of materials. Thus, in most of the cases, changing the material of study requires synthesizing the same peptide with a distinct anchor, resulting in expensive, time-consuming and repetitive procedures.

a)

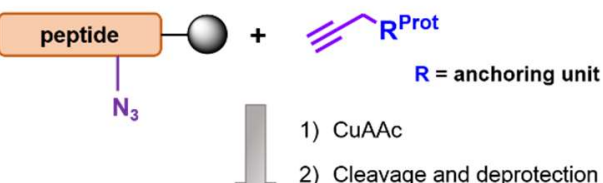

2) Cleavage and deprotection

b)

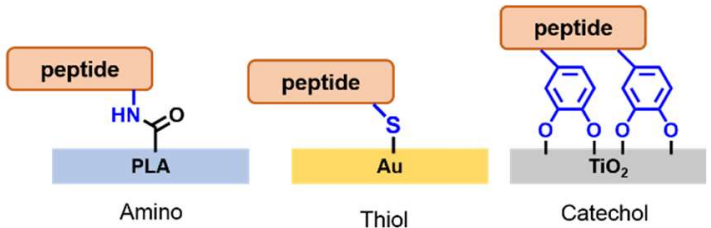

Fig. 1 a) General overview of the click-based solid-phase synthetic strategy used to prepare functionalized peptidic scaffolds. b) Schematic representation of biomaterials functionalized with the multifunctional peptide incorporating suitable anchoring units (amino, thiol and catechol). 
a)
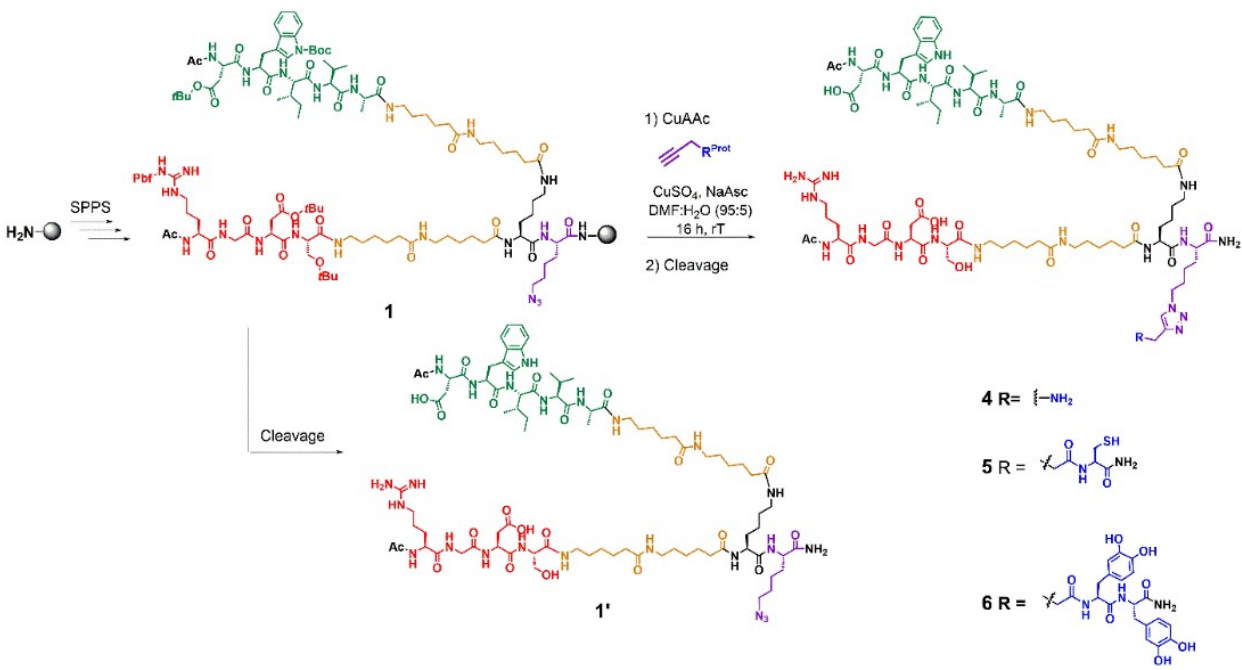

b)

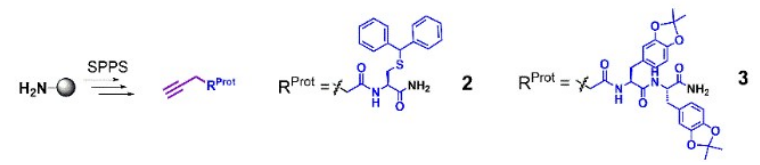

Fig. 2 Schematic representation of the click-based solid-phase synthetic strategy. a) Synthesis of the peptidic scaffolds containing the different anchoring units and b) synthesis of the protected anchoring units $\mathbf{2}$ and $\mathbf{3}$

To address these drawbacks, here we report on a novel and versatile click-based solid-phase synthetic strategy to prepare peptidic scaffolds for functionalizing a large variety of biomaterials (Fig. 1). The use of copper(I)-catalysed azide-alkyne cycloaddition reaction (CUAAc) on solid-phase allows the preparation of peptide mimetics containing specific anchoring units in a selective and stereospecific manner. ${ }^{19,20}$

As a proof of concept, we selected a biomimetic multifunctional peptide, recently developed by $\mathrm{us}^{21}$ and focused on the incorporation of three different anchoring groups (thiol, catechol and amino) in solid-phase. To this end, suitable functional groups were incorporated in both moieties, i.e. an azide group in the peptide and terminal alkynyl groups in the anchoring units, thus allowing their efficient and modular assembly by click chemistry.

The general synthetic strategy is presented in Fig. 2. The multifunctional peptidic scaffold combining the integrin-binding motif Arg-Gly-Asp-Ser (RGDS), which promotes cell adhesion, ${ }^{5,14}$ with the Asp-Trp-Ile-Val-Ala (DWIVA) sequence, which is involved in the BMP-2-BMPR-1 interaction and displays osteogenic potential. ${ }^{22}$ The target multifunctional RGDS-DWIVA peptide was assembled by $\mathrm{Fmoc} / \mathrm{tBu}$ solid-phase peptide synthesis (SPPS), following previously optimized protocols (Fig. 2a). ${ }^{23,24}$ First, Fmoc-L-Lys $\left(\mathrm{N}_{3}\right)-\mathrm{OH}$ was incorporated to Rink Amide $\mathrm{MBHA}$ resin to provide with an azido group the peptidic scaffold, a crucial element required to build the first component of the functionalization toolkit. Next, after the incorporation of the orthogonally-protected Fmoc-L-Lys(Alloc)-OH residue, which acts as a branching point, the RGDS sequence was elongated. To ensure an optimal separation between the two peptide binding motifs, and the material surface, two 6aminohexanoic acid units were introduced. ${ }^{19,25}$ The Alloc group was then removed using a catalytic amount of palladium, which allowed the second branch (DWIVA) to be subsequently assembled. In order to avoid undesired reactions, the $\mathrm{N}$ terminus of both branches was acetylated. The detailed synthetic protocols can be found in the Supporting Information.

Next, we focused on the preparation of the three alkynylderivatized anchoring units (Fig. 2b). While amino groups could be easily introduced using the commercially available propargylamine, the required thiol (2) and catechol (3)-based anchoring moieties were synthesized by SPPS on Fmoc-SieberPS since this solid support allows the side-chain protecting groups to be preserved after the cleavage. This was particularly important for building block 2 to avoid possible side-reactions of the thiol function during the CuAAc reaction. ${ }^{26}$ For the synthesis of 2, Fmoc-L-Cys(Dpm)-OH was first incorporated to the resin, followed by the coupling of 4-pentynoic acid. The diphenylmethyl (Dpm) group is fully compatible with the mild acidic conditions required for the cleavage from Sieber resin.

The same procedure was followed for the synthesis of 3: two suitably-protected L-3,4-dihydroxyphenylalanine (DOPA) residues and 4-pentynoic acid were sequentially incorporated on the Sieber resin by standard SPPS. Finally, both protected building blocks ( $\mathbf{2}$ and $\mathbf{3}$ ) were isolated with high purity from the solid support by using a mild acidic treatment (3\% TFA in DCM), and fully characterized by ${ }^{1} \mathrm{H}$ and ${ }^{13} \mathrm{C}$ NMR and HR ESI-MS (Fig. S4, S6 and S7).

CuAAc was performed on solid-phase using the methodology described by Rovira and co-workers. ${ }^{27}$ The reaction was first investigated by reacting the resin-bound azido-functionalized peptide platform 1 with propargylamine in the presence of $\mathrm{CuSO}_{4}$ and sodium ascorbate in $\mathrm{DMF} / \mathrm{H}_{2} \mathrm{O}$ 95:5 ( $\mathrm{v} / \mathrm{v})$ at different reaction times ( 2 and $16 \mathrm{~h})$. After acid deprotection (TFA/TIS/ $\mathrm{H}_{2} \mathrm{O}, 95: 2.5: 2.5$ ), HPLC analysis revealed the formation of the expected peptide platform 4, the analogue containing an amino group as anchoring unit, with $50 \%$ conversion at long times (Fig. S1). In order to improve the 
quality of the crude and the conversion yield, the resin was previously washed with sodium diethyldithiocarbamate to remove any $\mathrm{Pd}$ traces from the Alloc removal step. As shown in Fig. S2, the click reaction was improved, indicating that Pd traces were interfering with CuAAc. This optimized procedure was followed for the attachment of building blocks $\mathbf{2}$ and $\mathbf{3}$ to the resin-bound azido-functionalized peptide $\mathbf{1}$ to provide thiol-modified peptide 5 and DOPA-modified peptide $\mathbf{6}$, respectively. In both cases, final side-chain deprotection and cleavage from the resin was performed by treatment with TFA in the presence of suitable scavengers (dithiothreitol (DTT) was added in the case of $\mathbf{5}$ to prevent side reactions involving the thiol function, such as oxidation (Fig. S3)). Compounds 4-6 were purified by reversed-phase HPLC and characterized by HR ESIMS.

In order to validate the toolkit strategy, polylactic acid (PLA), gold and titanium were chosen as model biomaterial substrates to be functionalized via amino (4), thiol (5) and DOPA (6) anchoring groups, respectively. The non-modified peptide $\mathbf{1}^{\prime}$ was chosen as control to exclude unspecific interactions with the surfaces. The specificity of the peptides was first verified by XPS (Fig. 3). As expected, the incorporation of DOPA on RGDSDWIVA peptide (compound 6) yielded the highest binding to titanium samples, as evidenced by the increase in the percentage of the nitrogen signal (\% N1s), compared to control titanium and the rest of the peptides (Fig. 3 and Table S1). The grafting density obtained was of $77.4 \pm 2.1 \mathrm{pmol} / \mathrm{cm}^{2}$ (details can be found in the Supplementary Information). This effect was attributed to the capacity of catechol groups to establish coordinative interactions with $\mathrm{TiO}_{2}$, forming very stable bonds. ${ }^{18,28-30}$ In contrast, the presence of a thiol anchor (compound 5), shifted the affinity of the peptide towards gold substrates, due to the formation of a highly stable covalent Au$\mathrm{S}$ bond. ${ }^{31}$ In this case, the other peptides showed moderate to high values of attachment, which may be due to electrostatic interactions (peptide 4),32 binding between gold and the catechol group, either via the $o$-hydroxy or its aromatic ring (peptide 6), ${ }^{29,33,34}$ or simply unspecific physical adsorption (peptide $\mathbf{1}^{\prime}$ ). Nonetheless, the \% of N1s reached for these peptides was significantly lower than that obtained via the thiol$\mathrm{Au}$ interaction (Fig. 3 and Table S1). Finally, peptide 4 was coupled to PLA. To this end, the surfaces were $\mathrm{O}_{2}$-activated to render free carboxyl groups and promote electrostatic interactions with 4 . This procedure significantly increased the amount of nitrogen on the surfaces compared to unmodified PLA; however, the rest of peptides, including control 1', yielded similar values of attachment.

Subsequently, the results obtained from XPS measurements were further corroborated by a cell adhesion assay with human mesenchymal stem cells (MSCs). On the basis of the well-known biological activity of the RGD/DWIVA sequences, 5,14,22 it was anticipated that MSC adhesion would be positively influenced on the surfaces functionalized with this molecule. In particular, the number of adherent cells, their spreading (projected area) and morphology were analysed (Fig. 4 and S8).

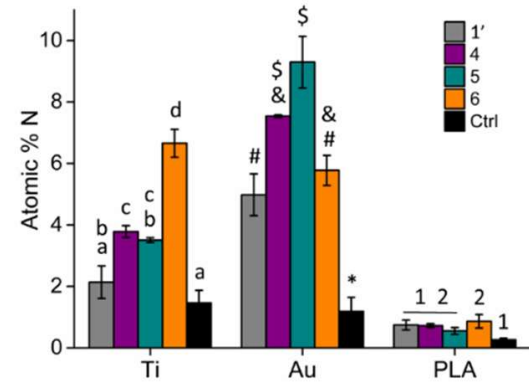

Fig. 3 Atomic percentages (\%) of $N$ 1s measured by X-ray photoelectron spectroscopy (XPS). Different letters, symbols and numbers denote statistically significant difference $(p<0.05)$ between Ti, Au and PLA samples, respectively.

As expected, the functionalization of titanium with peptide 6 yielded the highest number and spreading of adherent cells (Fig. 4a and 4b). These effects are clearly visible in Fig. 4c, especially when compared to uncoated surfaces. The functionalization of titanium with the other peptides resulted in significantly lower values of both cell number and area. Cell adhesion on Au samples was also in accordance with the XPS results. Indeed, non-functionalized Au surfaces exhibited a very low number of attached cells, which were totally rounded (Fig. 4). Interestingly, when functionalizing Au samples with the peptides, the number of adherent cells significantly increased in all cases (Fig. 4a), in clear correlation with the affinity observed for different anchoring chemistries to gold (Fig. 3). Yet, the highest number of attached cells was observed on Au samples functionalized with $\mathbf{5}$, thereby indicating the optimal binding of the peptide to the surface via the thiol-gold bond. The projected area on such samples was also significantly enhanced, and cells were shown to develop well defined actin filaments (Fig. $4 \mathrm{~b}$ and 4c). Finally, our toolkit approach was also validated on polymeric surfaces. In particular, cell adhesion was significantly improved on PLA films functionalized with peptide 4 (Fig. 4); this condition displayed the highest number and spreading of cells, regarding all the other conditions (Fig. 4a and 4b), and demonstrates the high specificity of amino groups towards activated PLA (i.e. negatively charged carboxyl groups). On PLA films coated with either thiol- or DOPA-containing peptides (5 and 6 , respectively), the number and area of attached cells was also improved compared to PLA functionalized with $\mathbf{1}^{\prime}$ and PLA control (Fig. 4 and S8). Such enhancement was attributed to peptide adsorption on the surface via other (e.g. nonelectrostatic) mechanisms. Moreover, to improve the extent of surface functionalization with peptide 4 , the carboxyl groups of PLA were activated with carbodiimide chemistry to promote the formation of covalent amide bonds. Of note, this strategy significantly increased the amount of $\mathbf{4}$ bound to the surfaces (Fig. S9), further improving the adhesion of MSCs to PLA (Fig. S10).

In conclusion, we reported a synthetically efficient and versatile toolkit approach for the coating of biomaterials by multifunctional peptidic scaffolds with high affinity. Three different anchoring chemistries-catechol, thiol and aminowere developed to specifically functionalize titanium, gold and polylactic acid, respectively. Of note, the functionalization of 
these three biomaterials with our methodology enhanced the adhesion of human MSCs, thus validating the feasibility of the strategy and paving the way to the straightforward synthesis of multifunctional, substrate-specific bioactive molecules in a time-efficient manner.
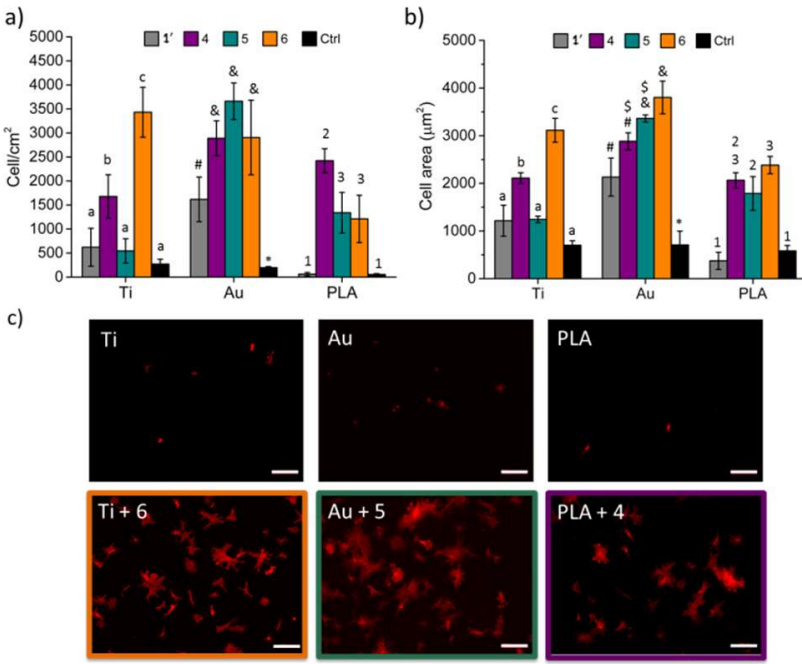

Fig. 4 MSCs biological characterization of functionalized surfaces ( $\mathrm{Ti}, \mathrm{Au}$ and PLA) A) Quantification of cell number and B) projected cell area after incubating the cells $6 \mathrm{~h}$ in serum-free medium. C) F-actin immunostaining of the nonfunctionalized surfaces (Ctrl) and $\mathrm{Ti}+\mathbf{6}, \mathrm{Au}+\mathbf{5}$ and PLA $+\mathbf{4}$ (scale bar $=200 \mu \mathrm{m}$ ). Different letters, symbols and numbers denote statistically significant difference $(p<0.05)$ between $\mathrm{Ti}, \mathrm{Au}$ and PLA samples, respectively.

\section{Conflicts of interest}

There are no conflicts to declare.

\section{Notes and references}

M. P. Lutolf and J. A. Hubbell, Nat. Biotechnol., 2005, 23, 47-55.

M. J. Dalby, A. J. García and M. Salmeron-Sanchez, Nat. Rev. Mater., , DOI:10.1038/natrevmats.2017.91. C. Mas-Moruno, B. Su and M. J. Dalby, Adv. Healthc. Mater., 2019, 8, 1801103. C. Hu, D. Ashok, D. R. Nisbet and V. Gautam, Biomaterials, 2019, 219, 119366.

5 C. Mas-Moruno, R. Fraioli, F. Rechenmacher, S. Neubauer, T. G. Kapp and H. Kessler, Angew. Chemie Int. Ed., 2016, 55, 7048-7067. M. Salmerón-Sánchez and M. J. Dalby, Chem. Commun., 2016, 52, 13327-13336.

C. Mas-Moruno, in Peptides and Proteins as Biomaterials for Tissue Regeneration and Repair, Elsevier, 2018, 73-100.

8 D. F. Williams, Biomaterials, 2011, 32, 4195-4197.

9 Y. Hirano and D. J. Mooney, Adv. Mater., 2004, 16, 17-25

10 X. Chen, P. Sevilla and C. Aparicio, Colloids Surfaces B Biointerfaces, 2013, 107, 189-197.

11 S. Bauer, P. Schmuki, K. von der Mark and J. Park, Prog. Mater. Sci., 2013, 58, 261-326.

12 R. Fraioli, S. Neubauer, F. Rechenmacher, B. M. Bosch, K.
Dashnyam, J. H. Kim, R. A. Perez, H. W. Kim, F. J. Gil, M. P. Ginebra, J. M. Manero, H. Kessler and C. Mas-Moruno, Biomater. Sci., 2019, 7, 1281-1285.

A. E. Rodda, L. Meagher, D. R. Nisbet and J. S. Forsythe, Prog. Polym. Sci., 2014, 39, 1312-1347.

U. Hersel, C. Dahmen and H. Kessler, Biomaterials, 2003, 24, 4385-4415.

F. Rechenmacher, S. Neubauer, J. Polleux, C. Mas-Moruno, M. De Simone, E. A. Cavalcanti-Adam, J. P. Spatz, R. Fässler and H. Kessler, Angew. Chemie Int. Ed., 2013, 52, 15721575.

J. C. Love, L. A. Estroff, J. K. Kriebel, R. G. Nuzzo and G. M. Whitesides, Chem. Rev., 2005, 105, 1103-1170.

S. Maity, S. Nir, T. Zada and M. Reches, Chem. Commun., 2014, 50, 11154-11157.

M. Pagel, R. Hassert, T. John, K. Braun, M. Wießler, B. Abe and A. G. Beck-Sickinger, Angew. Chemie - Int. Ed., 2016, 55, 4826-4830.

F. Rechenmacher, S. Neubauer, C. Mas-Moruno, P. M. Dorfner, J. Polleux, J. Guasch, B. Conings, H.-G. Boyen, A. Bochen, T. R. Sobahi, R. Burgkart, J. P. Spatz, R. Fässler and H. Kessler, Chem. - A Eur. J., 2013, 19, 9218-9223. V. Castro, H. Rodríguez and F. Albericio, ACS Comb. Sci., 2016, 18, 1-14.

L. Oliver-Cervelló, H. Martin-Gómez, L. Reyes, F. Noureddine, E. A. Cavalcanti-Adam, M.-P. Ginebra and C. Mas-Moruno, Adv. Healthc. Mater, 2020, 2001757. J.-Y. Lee, J.-E. Choo, Y.-S. Choi, J.-S. Suh, S.-J. Lee, C.-P. Chung and Y.-J. Park, Biomaterials, 2009, 30, 3532-3541. C. Mas-Moruno, R. Fraioli, F. Albericio, J. M. Manero and F. J. Gil, ACS Appl. Mater. Interfaces, 2014, 6, 6525-6536. M. Hoyos-Nogués, F. Velasco, M.-P. Ginebra, J. M. Manero, F. J. Gil and C. Mas-Moruno, ACS Appl. Mater. Interfaces, 2017, 9, 21618-21630.

C. Mas-Moruno, P. M. Dorfner, F. Manzenrieder, S. Neubauer, U. Reuning, R. Burgkart and H. Kessler, J. Biomed. Mater. Res. - Part A, 2013, 101 A, 87-97.

M. Góngora-Benítez, L. Mendive-Tapia, I. Ramos-Tomillero, A. C. Breman, J. Tulla-Puche and F. Albericio, Org. Lett., 2012, 14, 5472-5475.

A. Rovira, A. Gandioso, M. Goñalons, A. Galindo, A. Massaguer, M. Bosch and V. Marchán, J. Org. Chem., 2019, 84, 1808-1817.

H. Lee, N. F. Scherer and P. B. Messersmith, Proc. Natl. Acad. Sci., 2006, 103, 12999-13003.

H. Lee, S. M. Dellatore, W. M. Miller and P. B. Messersmith, Science (80-. )., 2007, 318, 426-430.

P. Kord Forooshani and B. P. Lee, J. Polym. Sci. Part A

Polym. Chem., 2017, 55, 9-33.

H. Häkkinen, Nat. Chem., 2012, 4, 443-455.

M. Ben Haddada, J. Blanchard, S. Casale, J.-M. Krafft, A. Vallée, C. Méthivier and S. Boujday, Gold Bull., 2013, 46, 335-341.

G. Lee, H. Lee, K. Nam, J.-H. Han, J. Yang, S. Lee, D. Yoon, K. Eom and T. Kwon, Nanoscale Res. Lett., 2012, 7, 608.

W. Zhang, H. Yang, F. Liu, T. Chen, G. Hu, D. Guo, Q. Hou, X. Wu, Y. Su and J. Wang, RSC Adv., 2017, 7, 32518-32527. 